\title{
Phenotype, function and T cell receptor repertoire of tumor-infiltrating lymphocytes in patients with pancreatic ductal adenocarcinoma
}

\author{
Isabel Poschke ${ }^{1 *}$, Michael Flossdorf ${ }^{1}$, Marta Faryna ${ }^{2}$, Frank Bergmann ${ }^{3}$, Jessica Hassel ${ }^{3}$, Oliver Strobel ${ }^{1}$, \\ Rienk Offringa'
}

From 30th Annual Meeting and Associated Programs of the Society for Immunotherapy of Cancer (SITC 2015) National Harbor, MD, USA. 4-8 November 2015

\section{Background}

Pancreatic ductal adenocarcinoma (PDAC) is a devastating disease with a median survival of only about two years even in the $20 \%$ of patients that present early enough to be eligible for surgical resection and adjuvant chemotherapy. With this urgent medical need in mind, we are exploring the use of adoptive $\mathrm{T}$ cell transfer in patients with PDAC, as this therapy has shown remarkable clinical success in patients with advanced melanoma.

\section{Results}

Despite the notion that, different from melanoma, PDAC is a non-immunogenic tumor, we observe at least moderate $\left(>150 \mathrm{CD}^{+}\right.$cells $\left./ \mathrm{mm}^{2}\right) \mathrm{T}$ cell infiltration in $60 \%$ of patient biopsies analyzed by immunohistochemistry $(\mathrm{n}=66)$. Flow cytometric analysis shows that tumorinfiltrating $\mathrm{T}$ lymphocytes (TILs) predominantly display an activated effector memory phenotype with signs of exhaustion or prolonged antigen exposure, such as high PD1 $(\mathrm{n} \geq 30)$.

Upon in vitro culture TILs can be expanded from $85 \%$ of PDAC patients $(n=96)$ and show growth capacity and phenotypes similar to melanoma patient TIL $(n=62)$. The majority of tested PDAC TIL cultures produce IFN- $\gamma$ in response to autologous tumor cells in an MHC-I dependent manner, but cross-reactivity and a low response magnitude are common observations.

In order to gain insight into the original tumor-reactivity of TILs before expansion, we studied the $\mathrm{T}$ cell receptor (TCR) repertoire by deep sequencing and made the following observations: the TIL TCR repertoire is i) distinct from the broad repertoire observed in the blood; ii) usually dominated by large $\mathrm{T}$ cell clones, possibly due to in situ expansion after tumor-antigen encounter; and iii) in most patients not maintained during the TIL expansion period, likely leading to a loss of important $\mathrm{T}$ cell clones and a shift in tumor-reactivity in the TILs available for patient treatment after in vitro expansion.

Deep sequencing of the TCR repertoire in combination with TCR cloning provides us with a tool to study TIL reactivity directly ex vivo and to 'rescue' TIL-TCRs with valuable reactivities that could be reintroduced in the form of genetically engineered $\mathrm{T}$ cells. Whether PDAC TILs are reactive towards shared antigens and/or mutation-derived neo-antigens is currently being investigated based on exome and RNA sequencing data from a subset of patients.

\section{Authors' details}

${ }^{1}$ German Cancer Research Center (DKFZ), Heidelberg, Germany. ${ }^{2} 3$ BioNTech Diagnostics GmBH, Mainz, Germany. ${ }^{3}$ Heidelberg University Hospital, Heidelberg, Germany.

Published: 4 November 2015

\section{doi:10.1186/2051-1426-3-S2-P44}

Cite this article as: Poschke et al:: Phenotype, function and T cell receptor repertoire of tumor-infiltrating lymphocytes in patients with pancreatic ductal adenocarcinoma. Journal for ImmunoTherapy of Cancer 2015 3(Suppl 2):P44. 